\title{
A reduced order model for Monte Carlo simulations of stochastic groundwater flow
}

\author{
Damiano Pasetto • Alberto Guadagnini · \\ Mario Putti
}

$2013 / 10 / 02$

Keywords Groundwater hydrology · Randomly heterogeneous transmissivity · Monte Carlo simulations · Reduced Order Model · Greedy Algorithm

1 Abstract We explore the ability of the greedy algorithm to serve as an effec2 tive tool for the construction of reduced order models for the solution of fully 3 saturated groundwater flow in the presence of randomly distributed transmis4 sivities. The use of a reduced model is particularly appealing in the context of 5 numerical Monte Carlo (MC) simulations that are typically performed, e.g., 6 within environmental risk assessment protocols. In this context, model order 7 reduction techniques enable one to construct a surrogate model to reduce the 8 computational burden associated with the solution of the partial differential 9 equation governing the evolution of the system. These techniques approximate 10 the model solution with a linear combination of spatially-distributed basis 11 functions calculated from a small set of full model simulations. The number 12 and the spatial behaviour of these basis functions determine the computational 13 efficiency of the reduced model and the accuracy of the approximated solu-

D. Pasetto

Institut National de la Recherche Scientifique,

Centre Eau Terre Environnement (INRS-ETE),

Université du Québec, G1K 9A9, Quebec City, Quebec, Canada

Now at Dipartimento di Matematica, University of Padova,

Via Trieste 63, 35121 Padova, Italy

E-mail: pasetto@math.unipd.it

A. Guadagnini

Dipartimento di Ingegneria Civile e Ambientale, Politecnico di Milano

Piazza L. Da Vinci 32, Milano, Italy

Department of Hydrology and Water Resources

University of Arizona, Tucson, Arizona 85721, USA

E-mail: alberto.guadagnini@polimi.it

M. Putti

Dipartimento di Matematica, University of Padova,

Via Trieste 63, 35121 Padova, Italy

E-mail: mario.putti@unipd.it 
14 tion. The greedy algorithm provides a deterministic procedure to select the

15 basis functions and build the reduced order model. Starting from a single ba-

16 sis function, the algorithm enriches the set of basis functions until the largest

17 error between the full and the reduced model solutions is lower than a prede-

18 fined tolerance. The comparison between the standard MC and the reduced

19 order approach is performed through a two-dimensional steady-state ground-

20 water flow scenario in the presence of a uniform (in the mean) hydraulic head

21 gradient. The natural logarithm of the aquifer transmissivity is modeled as a

22 second-order stationary Gaussian random field. The accuracy of the reduced

23 basis model is assessed as a function of the correlation scale and variance of

24 the log-transmissivity. We explore the performance of the reduced model in

25 terms of the number of iterations of the greedy algorithm, and selected met-

26 rics quantifying the discrepancy between the sample distributions of hydraulic

27 heads computed with the full and the reduced model. Our results show that the

28 reduced model is accurate and is highly efficient in the presence of a small vari-

29 ance and/or a large correlation length of the log-transmissivity field. The flow

30 scenarios associated with large variances and small correlation lengths require

31 an increased number of basis functions to accurately describe the collection

32 of the MC solutions, thus reducing significantly the computational advantages

33 associated with the reduced model.

\section{$34 \quad 1$ Introduction}

35 Modeling groundwater flow in natural aquifers requires coping with spatial het-

36 erogeneity of hydraulic properties, e.g., hydraulic conductivity and/or trans-

37 missivity. A proper characterization of these parameters is the key to, e.g., op-

38 timize water management and accurately predict transport of contaminants.

39 A deterministic approach to the solution of the flow problem is typically based

40 on an estimation of the spatial distribution of the hydraulic parameter fields

41 and the subsequent solution of the equations governing heads and fluxes. A

42 stochastic approach (e.g., $[1,2])$ describes parameters, such as aquifer trans-

43 missivity, as random fields with given probability distribution and aims at ren-

44 dering the probability distribution of state variables, such as hydraulic heads

45 and fluxes. A stochastic approach is appealing when sensitivity or uncertainty

46 analyses of hydraulic head distributions are required in the presence of in-

47 complete knowledge of the system parameters. It also enables one to embed

48 methodologies such as Markov chain Monte Carlo (MC) and ensemble Data

49 Assimilation for aquifer characterization under uncertainty (see e.g. [3, 4] and

50 references therein).

51 Approaches that have been employed to solve the stochastic groundwa-

52 ter flow equation include moment differential equations (MDE) formulations,

53 techniques based on partial differential equations satisfied by the probabil-

54 ity density function (pdf) of the state variable of interest, and the numerical

55 MC simulation framework. The idea underlying MDEs is the derivation of 
deterministic equations satisfied by the statistical (ensemble) moments of hydraulic head from the constitutive groundwater equation and on the basis of the knowledge of the (ensemble) moments of the system parameters, e.g., the transmissivity field. The expected value and covariance of the hydraulic heads are then numerically computed by way of recursive approximations of otherwise nonlocal MDEs (see e.g. [5, 6]). The MDE approach has been recently embedded within the context of Ensemble Kalman Filter based data assimilation procedures of groundwater flow [7]. Current formulations of MDEs cannot be easily extended to provide a complete characterization of the pdf of hydraulic heads in the presence of randomly variable transmissivities, because of the relatively complex formulation and prohibitive computational effort required to compute statistical moments of order larger than two.

An alternative approach, which has been developed mainly in the context of solute transport in randomly heterogeneous groundwater velocity fields (e.g., [8-11] and references therein), relies on the development of equations governing the space-time evolution of the pdf of solute concentrations. With the exception of a few special cases, the solution of these equations typically entails a series of approximations that are still limiting the direct application of the approach to practical aquifer scale environmental problems.

MC-based methods rely on the generation of multiple independent and identically-distributed realizations of the parameter fields driving groundwater flow. The corresponding solution of the flow equation yields a collection of realizations of hydraulic heads from which ensemble statistics can be evaluated. Although the implementation of MC methods is straightforward also in the presence of highly nonlinear models, the convergence of the empirical distribution to the underlying theoretical head probability distribution is generally slow and may require a large number of computationally-expensive solutions of the numerical model ([12] and references therein). For this reason, a routine application of MC simulations to real field-scale aquifer systems would significantly benefit from the development of a fast and accurate surrogate/reduced model for the computation of a large collection of hydraulic head realizations [13].

Techniques based on the Polynomial Chaos Expansion (PCE) approximation may constitute a viable framework to obtain such surrogate system models. PCE represents hydraulic head as a series of polynomials in terms of a given set of random parameters. These polynomials are orthonormal to the joint probability measure associated with the pdf of the uncertain system parameters ([14-17], and references therein). This expansion enables the efficient computation of the moments of hydraulic head and, eventually, its complete pdf. The spatially-distributed coefficients of the series are computed upon relying on the solution of the flow equation according to the Galerkin projection or the probabilistic collocation method (PCM) [18]. The number of elements to retain in the series expansion and evaluations of the original groundwater flow equation depends on the number of independent random parameters appearing in the flow equation. For this reason the PCE is typically applied only in the presence of a limited number of random parameters, an alternative being 
102 the reliance on an approximation (e.g., truncated Karhunen-Loève expansion)

103 of the (spatially-distributed) stochastic parameters.

104 Surrogate models developed within the context of Galerkin projection meth-

105 ods are of critical interest for the reduction of the computational cost related

106 to the numerical evaluation of the collection of hydraulic head realizations. To

107 achieve accurate and sizeable reduction, these surrogate models are built by

108 projecting the original model equations onto a set of basis functions calculated

109 from a limited number of solutions of the complete model of the system. This is

110 typically termed offline phase, the online phase being the ensuing application

111 of the surrogate model to form the ensemble. The model reduction procedure

112 is computationally advantageous when the dimension of the reduced model,

113 i.e., the number of employed basis functions, is considerably smaller than that

114 of the original model. Proper orthogonal decomposition (POD) [19, 20] and

115 reduced basis $(\mathrm{RB})[21,22]$ are two model order reduction approaches that

116 compute spatially-distributed basis functions within an offline procedure based

117 on the snapshot technique. This technique relies on a collection of a certain

118 number of full system model solutions, i.e., the solutions of the original model

119 equations obtained for selected observation times and/or parameter values.

120 POD performs a singular value analysis on the set of the snapshots. The prin-

121 cipal components associated with the rightmost singular values constitute an

122 optimal set of basis functions for the reproduction of the snapshots, in the

123 sense that any other set of the same size reproduces the snapshots with a

124 larger error. Several examples of application of POD to reduce the computa-

125 tional burden associated with deterministic groundwater flow problems can be

126 found in the literature. Siade et al. [23] use POD in the context of inverse mod-

127 eling to accelerate estimation of aquifer transmissivities with quasilinearization

128 and quadratic programming. Kaleta et al. [24] and van Doren et al. [25] de-

129 velop a reduced order model for the solution of the flow equations for reservoir

130 simulation and the corresponding adjoint system. The principal component

131 analysis is critical to remove redundant information when a large number of

132 snapshots is available. The number of snapshots and principal components

133 that one should employ to obtain a reduced model with a desired accuracy on

134 the solution depends critically on the target model and cannot be quantified a

135 priori. For example, Pasetto et al. [13] show that the application of the POD

136 methodology in the presence of a stochastic and spatially-distributed recharge

137 (which constitutes an additive noise for the flow equation) is strongly affected

138 by the variance and correlation length of the recharge term.

139 The RB approach (see, e.g. [22]) relies on the computation of the full sys-

140 tem model solution only for those snapshots that maximize the amount of

141 information to be embedded in the reduced model. In this case, the orthonor-

142 malized snapshots are directly selected as basis functions, thus circumventing

143 the need for a principal component analysis. To determine the dimension of

144 the reduced model, a validation set of parameter values is first considered;

145 new snapshots are then sequentially added to the reduced model until the full

146 model solutions employed in the validation set are reproduced within a given

147 level of accuracy. The procedure for the selection of the snapshots is based on 
148 the so-called greedy algorithm. Given a set of basis functions, a new snapshot 149 is computed by considering the parameter realization (selected amongst the 150 parameter realizations forming the validation set) that maximizes the discrep151 ancy between the full system model and the reduced model solutions. The 152 algorithm terminates when the maximum estimated error on each hydraulic 153 head solution of the validation set falls below a preselected tolerance. Since the 154 error between the reduced order and the full system model solutions cannot 155 be explicitly computed (because this requires the computationally expensive 156 full system model solution for all parameters in the validation set), the norm 157 of the residual is usually employed as a measure of the discrepancy between 158 the two solutions. Grepl and Patera [21] develop an a posteriori error bound 159 based on the computation of the residual to assess the accuracy of the reduced 160 model solution. This technique relies on an automatic procedure to establish 161 the basis functions that are required to achieve the reduction at a desired level 162 of accuracy. Pasetto et al. [26] demonstrate that the greedy algorithm is a 163 viable methodology to construct an accurate reduced model for the simulation 164 of groundwater flow in the presence of random transmissivity, when the latter 165 is described by a zonation approach. They show that the number of iterations 166 of the greedy algorithm, which coincides with the number of full system model 167 solutions, is determined by the error tolerance and the number of independent 168 random parameters (i.e., transmissivity zones) considered in the model.

169 Here, we study the implementation of the RB approach to construct a 170 reduced order model of steady-state groundwater flow driven by a randomly 171 distributed transmissivity field characterized by a log-normal probability den172 sity. The latter constitutes a multiplicative noise to the flow equation and still 173 represents a critical challenge in modern stochastic hydrogeology. We provide 174 a set of guidelines to establish the appropriate tolerance level to be set in the 175 greedy algorithm by considering a synthetic scenario representing a uniform 176 flow in the mean taking place within a two-dimensional bounded domain. The 177 convergence rate of the greedy algorithm is investigated as a function of the 178 correlation length and variance of the random transmissivity field. We explore 179 the relationship between the number of iterations of the greedy algorithm and 180 the reduced-model accuracy upon implementing the algorithm by considering 181 the explicit computation of the error on the basis of a large collection of MC 182 realizations. This allows circumventing inaccuracies related to the use of the 183 residual-based error estimations, which are only partially informative of the 184 head pdf stemming from MC simulations. The reliability of the heuristic crite185 ria underlying the application of the greedy algorithm is assessed in terms of 186 the maximum norm of the error between reduced order and full system model 187 results. Finally, we assess the accuracy of the reduced order model by compar188 ing the low order moments (ensemble mean and variance) and the empirical 189 probability distribution of nodal hydraulic heads resulting from a large set of $190 \mathrm{MC}$ simulations performed with the full system model and the reduced order 191 model subject to different values of the error tolerance. 


\section{Problem Setting}

193 We consider a fully saturated groundwater flow in a porous domain with random hydraulic properties, described by the stochastic equation:

$$
\begin{cases}-\nabla \cdot(T(\mathbf{x}, \omega) \nabla h(\mathbf{x}, \omega))=0, & \mathbf{x} \in S \\ h(\mathbf{x})=h_{D}(\mathbf{x}) & \mathbf{x} \in \partial S_{D} \subset \partial S \\ -T(\mathbf{x}, \omega) \nabla h(\mathbf{x}, \omega)=q_{N}(\mathbf{x}) & \mathbf{x} \in \partial S_{N} \subset \partial S\end{cases}
$$

where $\mathbf{x}$ is spatial-coordinate vector in the domain $S\left(S \subset \mathbb{R}^{d}, d=1,2\right.$, or 3), $\partial S$ is the boundary of the domain $S, h$ is hydraulic head, $\omega$ is a random sample in the space of outcomes $\Omega$, and $T$ is a randomly heterogeneous spatial transmissivity field. The functions $h_{D}$ and $q_{N}$ are the hydraulic heads and Darcy fluxes prescribed at the Dirichlet boundary $\partial S_{D}$ and at the Neumann boundary $\partial S_{N}$, respectively. We consider $T$ as a stationary stochastic process, characterized by a $\log$-normal distribution, i.e., $Y=\log T$ is a Gaussian random field, with uniform mean $\mu_{Y}$ and covariance function $C_{Y}$,

$$
C_{Y}(r)=\sigma_{Y}^{2} \rho_{Y}(r)
$$

where $r$ is separation distance (lag), and $\sigma_{Y}^{2}$ and $\rho_{Y}(r)$ are the variance and the correlation function of $Y$, respectively.

The probability space is discretized by means of a number $N_{\text {ens }}$ of MC samples. Let $\mathcal{Y}$ be the set of the independent random realizations of $Y$ employed in the MC approach, $\mathcal{Y}=\left\{Y^{(1)}, \ldots, Y^{\left(N_{e n s}\right)}\right\}$ and $\mathcal{T}=\left\{T^{(1)}, \ldots, T^{\left(N_{e n s}\right)}\right\}$ the corresponding transmissivities set. The numerical discretization of the flow problem is obtained by means of the Galerkin finite element method with piecewise linear elements on a triangular grid with $n$ nodes. Solving Eq. 1 for each element $T^{(i)} \in \mathcal{T}$ entails dealing with a high dimensional sparse linear system

$$
\mathbf{A}^{(i)} \mathbf{h}^{(i)}=\mathbf{b}
$$

where $\mathbf{A}^{(i)}$ is the stiffness matrix of dimension $n \times n$ associated with the realization $T^{(i)}, \mathbf{h}^{(i)}$ is the vector of hydraulic heads at the grid nodes and $\mathbf{b}$ is the vector accounting for the boundary conditions. Hereafter we refer to the solution of Eq. 3 as the full system model (FSM). Given the calculated collection of MC realizations $\left\{h^{(1)}, \ldots, h^{\left(N_{e n s}\right)}\right\}$, an ensemble moment $\mu$ of hydraulic head at grid node $x_{j}$ is approximated by its sample counterpart $\mu^{F S M}$ :

$$
\mu\left(h\left(x_{j}\right)\right)=\int_{\mathbb{R}} \phi(h) p_{h}(h) d h \approx \mu^{F S M}\left(h_{j}\right)=\frac{1}{N_{\text {ens }}} \sum_{i=1}^{N_{\text {ens }}} \phi\left(h_{j}^{(i)}\right),
$$

$\phi$ and $p_{h}$ being an integrable function in probability space and the pdf of $h$ at node $x_{j}$, respectively. 


\section{Reduced Order Model and Greedy Algorithm}

The reduced order model is constructed relying on a Galerkin projection technique, which is at the basis of both the RB and POD methodologies. The vector of nodal hydraulic heads is approximated by the sum of a mean head field, $\mathbf{h}^{(0)}$ and a linear combination of $N_{B F}$ spatially-distributed basis functions $\mathbf{p}_{j}$ :

$$
\mathbf{h}^{(i)} \approx \tilde{\mathbf{h}}^{(i)}=\mathbf{h}^{(0)}+\sum_{j=1}^{N_{B F}} a_{j}^{(i)} \mathbf{p}_{j}=\mathbf{h}^{(0)}+\mathbf{P} \mathbf{a}^{(i)} .
$$

Here, the vector of the coefficients $\mathbf{a}^{(i)}=\left\{a_{1}^{(i)}, \ldots, a_{N_{B F}}^{(i)}\right\}$ depends on the random realization $T^{(i)}$, and $\mathbf{P}$ is the matrix whose columns are the basis functions $\mathbf{p}_{j}$. The mean head field $\mathbf{h}^{(0)}$ may be approximated in different ways. For instance, one can employ the solution of Eq. 1 where $T$ is replaced by the geometric mean of the transmissivity field (which is a relatively robust approximation of the mean head in the absence of forcing terms such as pumping). Alternatively, the solution of the approximated moment equations satisfied by the (ensemble) mean head can be used (see [5]). The coefficients $\mathbf{a}^{(i)}$ are computed in the reduced dimension, i.e., they are the solution of a linear system of dimension $N_{B F} \times N_{B F}$ (instead of being the solution of the $n \times n$ FEM system approximating the original groundwater flow model) obtained by projecting Eq. 3 onto the space generated by the $N_{B F}$ basis functions $\mathbf{p}_{j}, j=1, \ldots, N_{B F}$. Hence, according to the Galerkin method, we substitute $\mathbf{h}^{(i)}$ with its approximation $\tilde{\mathbf{h}}^{(i)}$ in Eq. 3 and orthogonalize the residual with respect to the basis functions $\mathbf{P}$, obtaining the Reduced Order Monte Carlo model (ROMC):

$$
\tilde{\mathbf{A}}^{(i)} \mathbf{a}^{(i)}=\tilde{\mathbf{b}}^{(i)}
$$

where $\tilde{\mathbf{b}}^{(i)}=\mathbf{P}^{T} \mathbf{b}-\mathbf{P}^{T} \mathbf{A}^{(i)} \mathbf{h}^{(0)}$ and $\tilde{\mathbf{A}}^{(i)}=\mathbf{P}^{T} \mathbf{A}^{(i)} \mathbf{P}$ is a symmetric full matrix with dimension $N_{B F} \times N_{B F}$.

Solving Eq. 6 is computationally more advantageous than solving the FSM when the number of basis functions is significantly smaller than the number of grid nodes, i.e. $N_{B F}<<n$. This is balanced by the requirement that enough basis functions of sufficient quality should be employed to guarantee the accurate reproduction of the empirical pdf evaluated from the MC realizations. Thus, the number of basis functions employed to achieve the reduction and their selection procedure characterize the reduced order model. Note that the reduction methodology can also be applied jointly with techniques that accelerate the convergence of the MC method, such as sparse grids, sparse approximation, polynomial chaos expansion, latin hypercube, or multilevel MC (see e.g., [27]).

The POD and RB approaches employ basis functions extracted from a prescribed number of FSM solutions, which are termed snapshots.

In the POD method the snapshot technique [13] relies on collecting a number $N_{\text {snap }}$ of FSM solutions and performing a principal component analysis of 
these snapshots. Then, the set of basis functions corresponds to the $N_{B F}$ principal components associated with the rightmost singular values and provides the best approximation of the snapshots according to a predefined criterion. The number of snapshots, the criteria for their selection, and the number of principal components to use in the reduction process are (in principle) arbitrary and strongly affect the quality of the reduction.

The RB method employs a different approach: the snapshots are computed using a greedy algorithm and are directly orthonormalized to form the set of basis functions used in the reduction. The greedy algorithm [28] is a deterministic methodology that enables one to obtain a set of suitable snapshots for the RB technique. The main advantage of this procedure with respect the POD method is that it requires only the collection of FSM solutions that are essential for the computation of the basis function set.

The greedy algorithm proceeds iteratively until some suitable metric quantifying the errors between the ROMC and FSM solutions is below a given tolerance $\tau$. At each iteration the scheme augments the set of the basis functions with the realization associated with the largest error between the full and reduced model solutions. The idea underlying this choice is that the information content embedded in such a realization is not included in the current set of basis functions. Since the computation of the error is costly as it requires the knowledge of the FSM solution, the RB approach employs an a posteriori error estimation to assess the accuracy of the reduced model. If the error estimation is larger then the tolerance $\tau$, the reduced model is enriched with a new basis function, otherwise the algorithm terminates. The a posteriori error estimation is typically based on the computation of the residual associated with the reduced model solution to preserve the computational efficiency of the algorithm.

Here, we analyze the convergence properties of the greedy algorithm when applied to the MC solution of the stochastic PDE in Eq. 1. As noted in the introduction, a non accurate evaluation of the error estimate may lead to an inefficient reduced model. For this reason, our implementation of the greedy algorithm in the RB context relies on the exact computation of the errors instead of considering residual-based error estimates (as described, e.g., in [22]). At each $k^{\text {th }}$-iteration of the greedy algorithm we compute the ROMC solutions $\left\{\tilde{\mathbf{h}}_{k}^{(1)}, \ldots, \tilde{\mathbf{h}}_{k}^{\left(N_{\text {ens }}\right)}\right\}$ using $N_{B F}=k$ basis functions, $\mathbf{P}_{k}=\left[\mathbf{p}_{1}, \ldots, \mathbf{p}_{k}\right]$. We consider the relative error measure defined as:

$$
\epsilon_{k}^{(i)}=\frac{\left\|\mathbf{h}^{(i)}-\tilde{\mathbf{h}}_{k}^{(i)}\right\|_{\infty}}{\left\|\mathbf{h}^{(i)}\right\|_{\infty}}
$$

i.e., $\epsilon_{k}^{(i)}$ is the largest nodal error norm calculated between the $i^{t h}$-solution of the FSM and the corresponding ROMC solution normalized by the largest head value of realization $i$. Let $g_{1}, \ldots, g_{k}$ be the indices of the head realizations employed as snapshots in the $k$-th iteration of the greedy algorithm. If the largest value amongst the errors $\left\{\epsilon_{k}^{(1)}, \ldots, \epsilon_{k}^{\left(N_{e n s}\right)}\right\}$ is smaller than the predefined tolerance $\tau$, then the algorithm terminates. Otherwise, the set of basis 
308 functions is enriched by employing the FSM solution that is associated with 309 the largest value of the relative error $\epsilon_{k}^{(i)}$. In other words, at iteration $k+1$ 310 we add the FSM realization characterized by the largest error norm, $\mathbf{h}^{\left(g_{k+1}\right)}$ 311 to the set of previously calculated snapshots, where

$$
g_{k+1}=\underset{1 \leq i \leq N_{e n s}}{\arg \max } \epsilon_{k}^{(i)}
$$

313 It follows that $\mathbf{p}_{k+1}$ is the orthonormalization of $\mathbf{h}^{(0)}-\mathbf{h}^{\left(g_{k+1}\right)}$ with respect 314 to the set of the previously calculated basis functions, $\left\{\mathbf{p}_{1}, \ldots, \mathbf{p}_{k}\right\}$. The ini315 tialization of the greedy algorithm is performed by setting $k=0, \tilde{\mathbf{h}}_{0}^{(i)}=\mathbf{h}^{(0)}$, 316 and

$$
g_{1}=\underset{1 \leq i \leq N_{\text {ens }}}{\arg \max } \epsilon_{0}^{(i)}, \quad \mathbf{p}_{1}=\frac{\mathbf{h}^{(0)}-\mathbf{h}^{\left(g_{1}\right)}}{\left\|\mathbf{h}^{(0)}-\mathbf{h}^{\left(g_{1}\right)}\right\|_{2}} .
$$

Algorithm 1 illustrates this methodology.

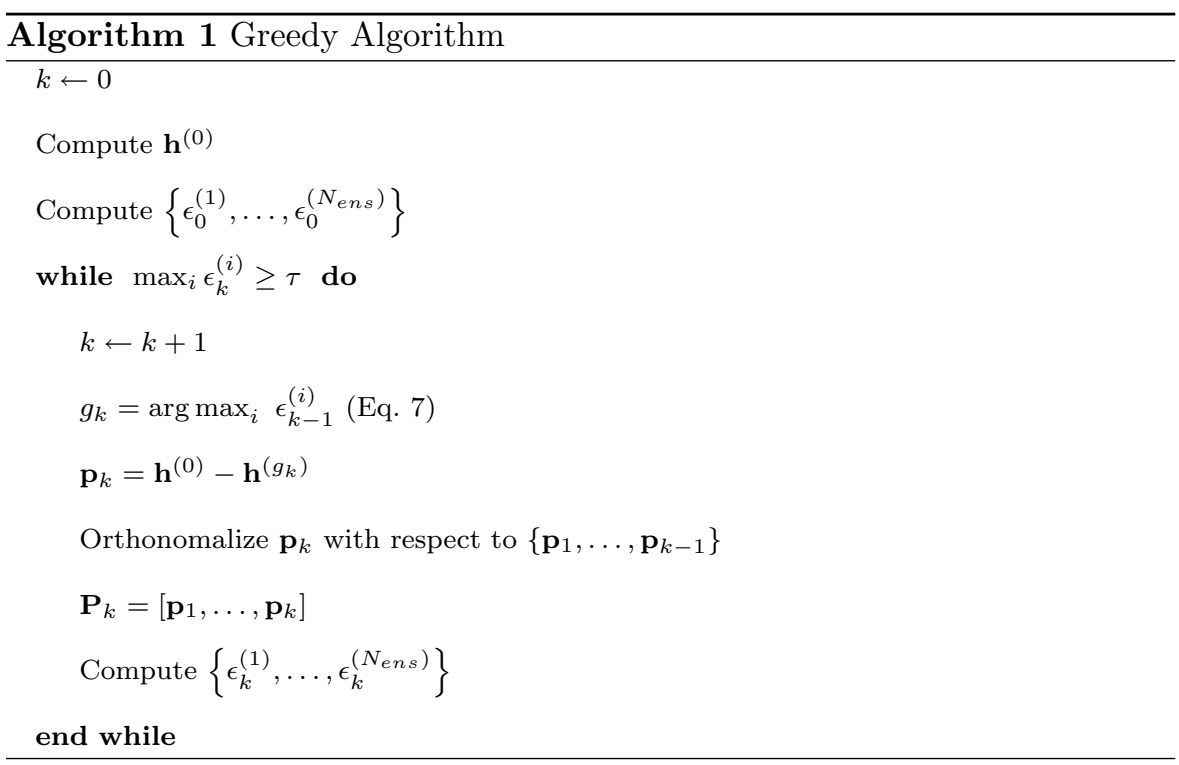

Several techniques are available in the literature to reduce the computational cost associated with the greedy algorithm, as detailed in the following. Since the FSM solutions are typically not available, the norm of the residual term $\mathbf{r}_{k}^{(i)}$,

$$
\mathbf{r}_{k}^{(i)}=\mathbf{b}-\mathbf{A}^{(i)} \tilde{\mathbf{h}}_{k}^{(i)}
$$

is usually employed for the evaluation of the error $\epsilon_{k}^{(i)}$ in Eq. 7 (see, e.g., [21, 26]). The evaluation of the residual in Eq. 9 requires only the computation of the FSM solution of the realizations that maximize the norm of the residual. 
Note that the norm of the residual can be computed in the reduced order space when $A^{(i)}$ is expressed as a linear combination of $N_{m}<<n$ matrixes, i.e.

$$
\mathbf{A}^{(i)}=\sum_{j=1}^{N_{m}} c_{j}^{(i)} B^{(j)},
$$

360 We solve Eq. 1 in the two-dimensional domain $S$ depicted in Fig. 1, which

361 has a rectangular shape of length $L_{x}=18$ and $L_{y}=8$ units in the $x$ and $y$

where $\xi_{j}^{(i)}$ is a point in the interval $\left[h_{j}^{(i)}, \tilde{h}_{j}^{(i)}\right]$ that satisfies the mean value theorem and $C_{j}=\max _{i}\left|\phi^{\prime}\left(h_{j}^{(i)}\right)\right|\left\|\mathbf{h}^{(i)}\right\|_{\infty}$. For example, one can note that $C_{j}=\max _{i}\left\|\mathbf{h}^{(i)}\right\|_{\infty}$ or $2 \max _{i}\left|h_{j}^{(i)}\right|\left\|\mathbf{h}^{(i)}\right\|_{\infty}$ for the first and second moment, respectively. Eq. 11 guarantees that the sample moments that are computed through the ROMC converge to the FSM moments when $\tau$ tends to zero. We also note that the error on the moments (Eq. 11) is bounded by the average nodal error. The latter can be, in turn, significantly smaller than the maximum nodal error, and can be used as a termination condition for the greedy algorithm.

\section{Numerical Example}




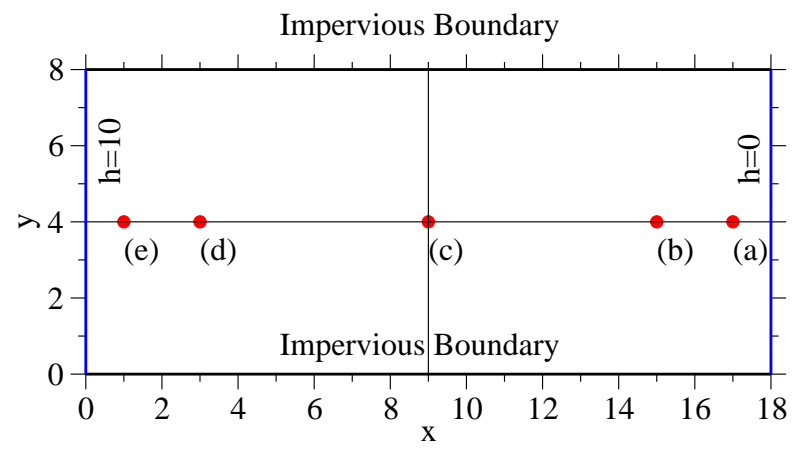

Fig. 1 Two-dimensional domain, $S$, employed in the numerical simulations. Location of control points considered in Fig. 7 is reported.

directions, respectively (here and in the following all quantities are given in consistent units). Prescribed heads of 10 and 0 are imposed at the left and right boundaries, respectively. Impervious boundary conditions are set at the top and bottom boundaries. The domain is discretized into $40 \times 90$ square cells, for a total of 3731 nodes.

The stationary log-transmissivity field, $Y$, is characterized by a normal 368 probability distribution with $\mu_{Y}=0$ and exponential isotropic covariance 369 function,

$$
C_{Y}(r)=\sigma_{Y}^{2} \exp \left(-\frac{r}{\lambda}\right)
$$

where $\lambda$ is the correlation length of $Y$. We analyze 24 test cases characterized by all the combinations of the following values of variance and relative domain size $L_{x} / \lambda$ :

$$
\sigma_{Y}^{2}=\{0.1,0.2,0.5,1.0\} ; \quad \frac{L_{x}}{\lambda}=\{9,4.5,3,2.25,1.8,1.5\}
$$

For each of these cases we generate $10^{4}$ independent MC realizations of the logtransmissivity field through the sequential Gaussian software HYDRO_GEN [29]. We then solve the FSM equation (Eq. 3) for each transmissivity field and compute the empirical distribution of the hydraulic head at the grid nodes.

Algorithm 1 is employed for the construction of the reduced order model. 380 We test four threshold values for the error tolerance, $\tau=\{8 \%, 6 \%, 4 \%, 2 \%\}$. 381 For each of these tolerances we compute the collection of ROMC heads and 382 compare their empirical pdf against the one obtained by way of the FSM 383 realizations. 


\section{Results and discussion}
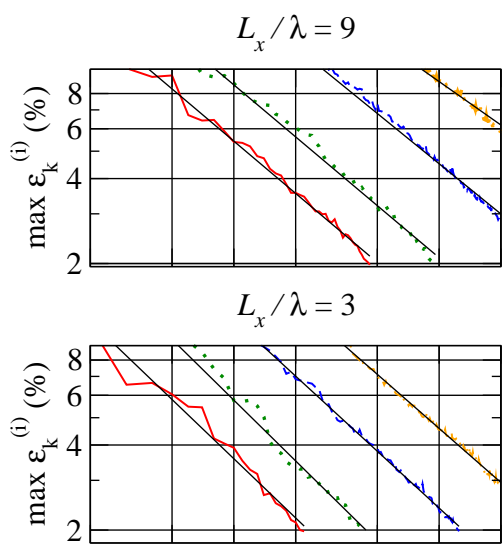

$L_{x} / \lambda=1.8$
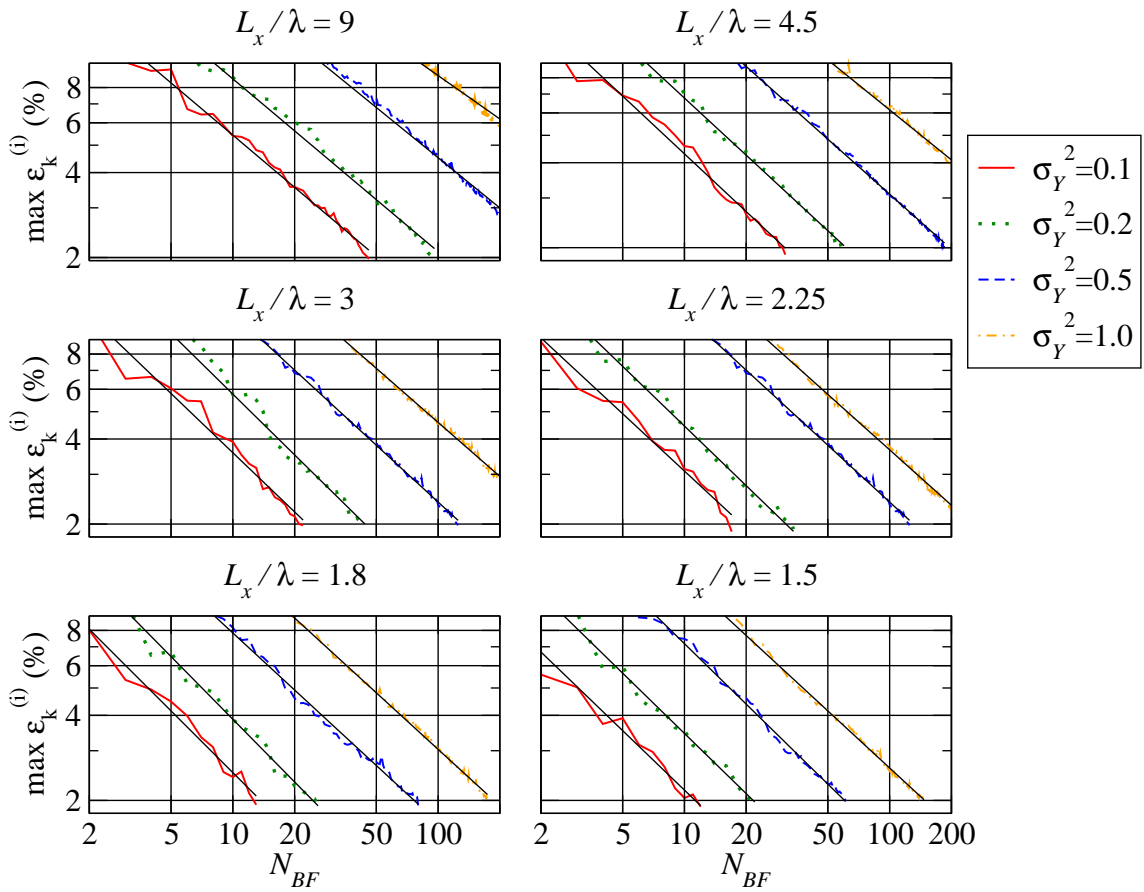

Fig. 2 Convergence of the maximum relative error $\epsilon_{k}^{(i)}$ with the number of basis functions

$N_{B F}$ employed in the reduced order model at each iteration of Algorithm 1. Results are illustrated for all 24 scenarios of log-transmissivity considered.

The number of iterations required to attain convergence of the greedy algorithm depends on the error tolerance $\tau$ and on the spatial variability of the random realizations of $h$. Realizations associated with large variability (both in space and in the ensemble sense) require an increased number of basis functions (i.e., of FSM solutions) to obtain approximation errors smaller than the tolerance $\tau$.

Fig. 2 depicts (in log-log scale) the convergence of the maximum nodal error $\epsilon_{k}^{(i)}$ as a function of the number of iterations of the greedy algorithm for the 24 test cases analyzed. For convenience, a maximum number of 200 iterations is set to terminate the algorithm. On one hand, we note that after 200 iterations the maximum error is still larger than the prescribed error tolerance when 


\begin{tabular}{l|cccc|cccc} 
& \multicolumn{7}{|c|}{$m$} & \multicolumn{5}{c}{$c$} \\
\hline$\sigma_{Y}^{2}$ & 0.1 & 0.2 & 0.5 & 1.0 & 0.1 & 0.2 & 0.5 & 1.0 \\
\hline$L_{x} / \lambda=9$ & -0.61 & -0.61 & -0.59 & -0.51 & 0.223 & 0.351 & 0.685 & 0.935 \\
$L_{x} / \lambda=4.5$ & -0.67 & -0.67 & -0.64 & -0.58 & 0.205 & 0.318 & 0.609 & 0.921 \\
$L_{x} / \lambda=3$ & -0.69 & -0.71 & -0.66 & -0.63 & 0.177 & 0.299 & 0.519 & 0.854 \\
$L_{x} / \lambda=2.25$ & -0.67 & -0.70 & -0.66 & -0.65 & 0.145 & 0.223 & 0.519 & 0.733 \\
$L_{x} / \lambda=1.8$ & -0.72 & -0.73 & -0.67 & -0.66 & 0.133 & 0.211 & 0.367 & 0.646 \\
$L_{x} / \lambda=1.5$ & -0.69 & -0.70 & -0.71 & -0.67 & 0.108 & 0.176 & 0.370 & 0.574
\end{tabular}

Table 1 Values of the order of convergence $m$ and coefficient $c$ of Eq. 14 for the 24 test cases analyzed.

$\tau=2 \%$ for the test cases associated with large variances and large relative domain size (e.g., $L_{x} / \lambda=9,4.5,3,2.25$, and $\sigma_{Y}^{2}=1.0$ ). On the other hand, convergence is fast for settings with small relative domain size and variance (e.g., $L_{x} / \lambda=1.8,1.2$, and $\left.\sigma_{Y}^{2}=0.1,0.2\right)$ and the imposed error tolerance is obtained with less than 30 iterations. Fig. 2 also reports the regression lines that approximate the convergence rate of the algorithm. These results reveal that the convergence rate of the greedy algorithm is well approximated by a power-law model of the kind:

$$
\max _{i}\left(\epsilon_{k}^{(i)}\right) \approx c k^{m}
$$

Table 1 summarizes the estimated values of $c$ and $m$ for the 24 test cases. The order of convergence $m$ slightly oscillates about a mean value of -0.66 and lies within the range $[-0.73,-0.51]$. The coefficient $c$ displays an approximately linear dependence on the relative domain size $L_{x} / \lambda$ and variance, its values increasing from 0.108 in the case with $L_{x} / \lambda=1.5, \sigma_{Y}^{2}=0.1$ to 0.935 for $L_{x} / \lambda=9$, $\sigma_{Y}^{2}=1.0$.

These results may be explained by the observation that large correlation lengths of the $Y$ field (i.e., low values of $L_{x} / \lambda$ ) are associated with relatively smooth spatial variations of $h$. This implies that the ensemble variance of hydraulic heads tends to decrease with decreasing $L_{x} / \lambda$ for the flow condition considered. As a consequence, only few basis functions are required to describe the realizations of head, and the greedy algorithm converges more rapidly. A corresponding argument explains the behavior observed for the settings associated with low variance of $Y$. 


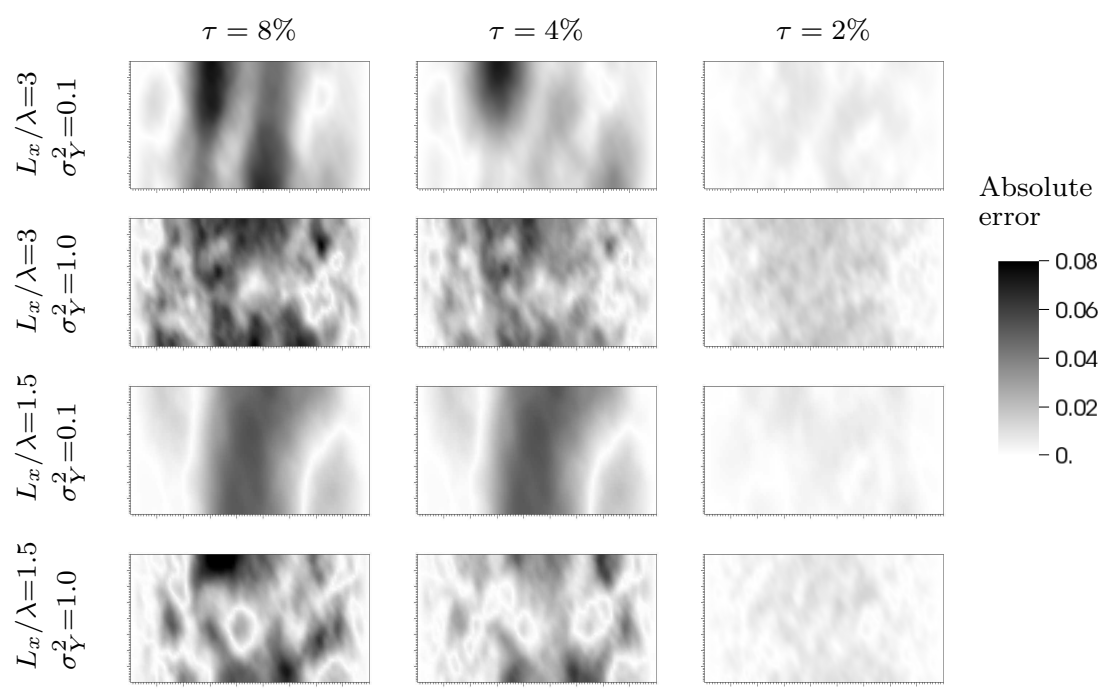

Fig. 3 Spatial distribution of the absolute error between the empirical variances of the hydraulic head realizations computed with the FSM and the ROMC. Results for four test cases (corresponding to all combinations of $L_{x} / \lambda=3,1.5$ and $\sigma_{Y}^{2}=1.0,0.1$ ) and three error tolerances $\tau=8 \%, 4 \%, 2 \%$. are shown.

\subsection{Estimation of head variance}

Here, we analyze the accuracy of the ROMC procedure for the approximation of the empirical variance of hydraulic heads. Figs. 3 and 4, respectively, depict the spatial distribution of the absolute and relative errors between the empirical variances computed via the FSM and the ROMC. Results are illustrated for four selected test cases (corresponding to all combinations of $L_{x} / \lambda=3,1.5$ and $\left.\sigma_{Y}^{2}=1.0,0.1\right)$ and three error tolerances $\tau=8 \%, 4 \%, 2 \%$. In agreement with Eq. 11, the error on the variance decreases throughout the domain as $\tau$ decreases, its values always being of the same order of magnitude as those of $\tau$ (see Fig. 3). This in turn implies that Eq. 11 can be considered as a conservative upper bound on the error performed by the ROMC on the computation of the second moment.

Note that the magnitude of the absolute errors depicted in Fig. 3 does not display large variations for a given error tolerance. This implies that $\tau$ controls the accuracy of the reproduction of the collection of MC realizations and their statistics. This result is consistent with Eq. 11, where it is seen that the error is independent of the correlation length and variance of $Y$. The generality of this conclusion within the range of parameters tested is supported by Fig. 5, where the boxplots of the distribution of the spatial nodal errors 


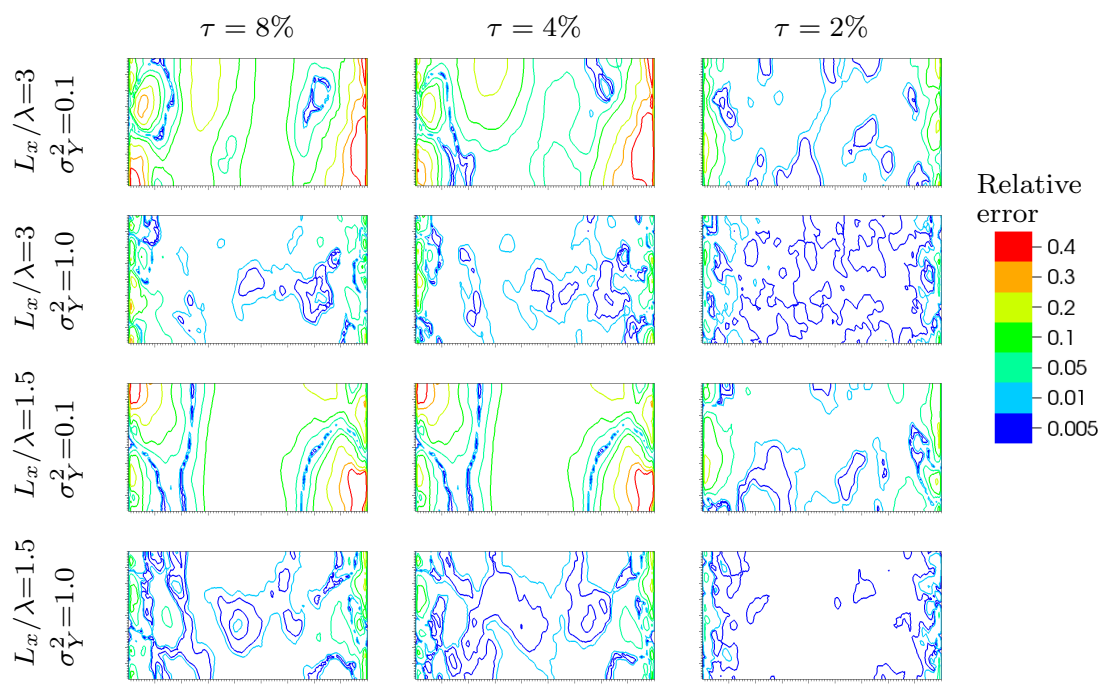

Fig. 4 Spatial distribution of the relative error between the empirical variances of the hydraulic head realizations computed with the FSM and the ROMC. Results for four test cases $\left(L_{x} / \lambda=3,1.5\right.$ and $\left.\sigma_{Y}^{2}=1.0,0.1\right)$ and three error tolerances $\tau=8 \%, 4 \%, 2 \%$. are shown.

on the head variance are shown for the complete set of test cases and the four error tolerances analyzed. Fig. 4 reveals that the largest relative nodal errors occur in the proximity of the Dirichlet boundary, i.e. where the head variance tends to vanish, while they are always smaller than the desired model accuracy at all other locations in the domain.

\subsection{Empirical distributions of nodal heads}

MC simulations are frequently employed (e.g., in environmental risk assessment protocols) to explore the behavior at the tails of the probability distribution. Here, we focus on the ability of the ROMC scheme to approximate the $10^{t h}$ - and $90^{t h}$-percentiles of nodal heads distributions, $q_{10}$ and $q_{90}$, respectively defined as:

$$
P\left(h<q_{10}\right)=10 \%, \quad P\left(h<q_{90}\right)=90 \%
$$

where $P$ indicates probability. Figs. 6 and 7 report the relative errors on the $q_{10}$ and $q_{90}$ for hydraulic heads at nodes located along the central vertical cross-section of the domain, where head variances are highest. By way of illustration and consistent with Figs. 3 and 4, the results are presented for the test cases associated with all combinations of $L_{x} / \lambda=3,1.5$ and $\sigma_{Y}^{2}=1.0,0.1$. 

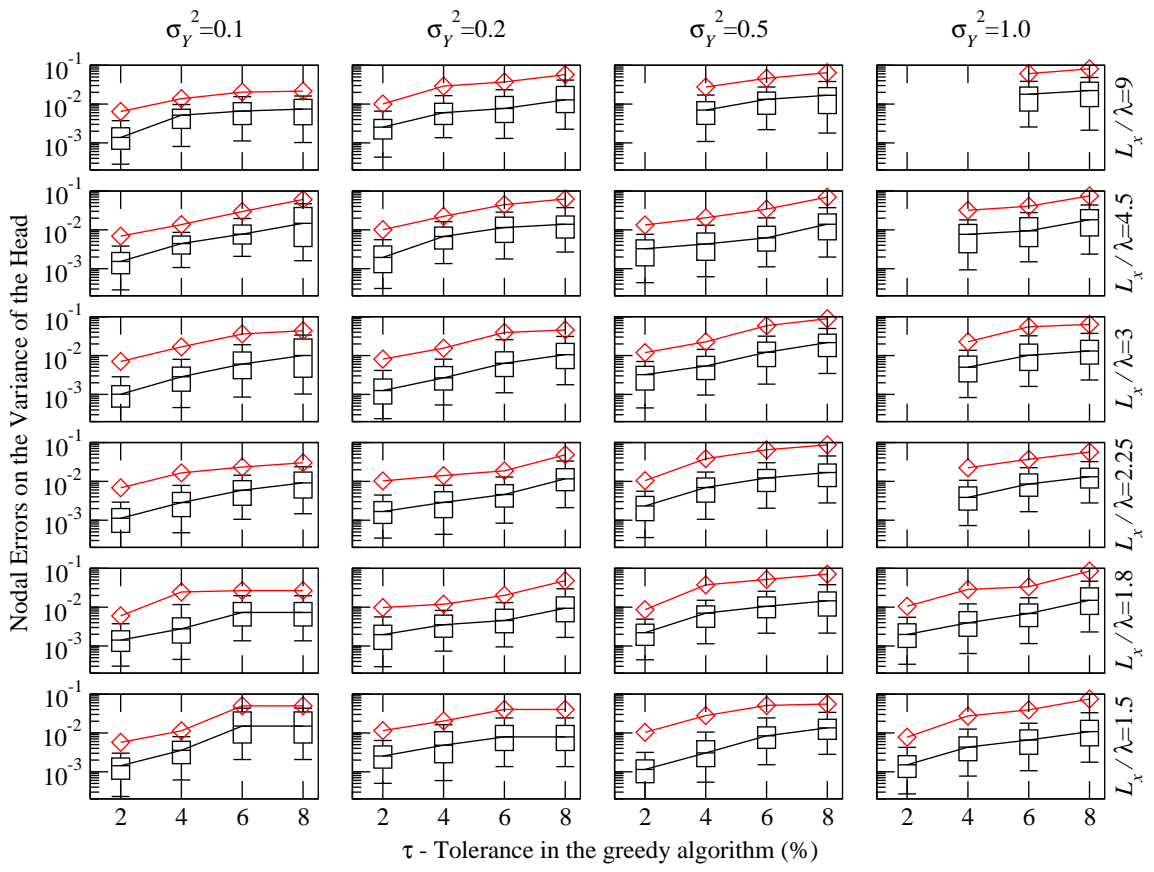

Fig. 5 Distribution of the nodal errors between the empirical hydraulic head variances computed with the FSM and the ROMC. Each boxplot depicts the $10^{t h}, 25^{t h}, 50^{t h}, 75^{t h}$ and $90^{\text {th }}$ percentile of the error distributions. The red curves represent the maximum error. Results of test cases that did not attain convergence are omitted.

The comparison of the calculated percentiles associated with the error tolerances $\tau=8 \%$ and $\tau=2 \%$, shows that the smallest error tolerance renders the best reproduction of the FSM percentiles. The maximum relative errors in both the $10^{t h}$ - and $90^{t h}$-percentiles are associated with $\sigma_{Y}^{2}=0.1$ and $\tau=8 \%$.

460 This is likely related to the small number of basis functions returned by the greedy algorithm in these two cases $\left(N_{B F}=3\right.$ for $L_{x} / \lambda=3$ and $N_{B F}=2$ for $\left.462 L_{x} / \lambda=1.5\right)$. We note that this effect is not visible for $\tau=2 \%$, where the er463 rors on the percentiles are of the same order of magnitude for all tested cases. 464 Similarly to what observed for the errors on the head variance (Section 5.3), 465 the magnitude of the relative errors on the percentiles are significantly smaller 466 than the error tolerances $\tau$ at most locations in the domain. Finally, Figs. 8 467 and 9 present a comparison between the complete empirical pdfs of the FSM 468 and ROMC head realizations at five selected points in the system. Results are 469 illustrated for two test cases $\left(L_{x} / \lambda=3, \sigma_{Y}^{2}=0.1\right.$ or 1.0) and error tolerances $470 \tau=8 \%, 2 \%$. The small differences between the pdfs, which have also been de471 tected in the analysis of the head variance and percentiles, do not appear to 

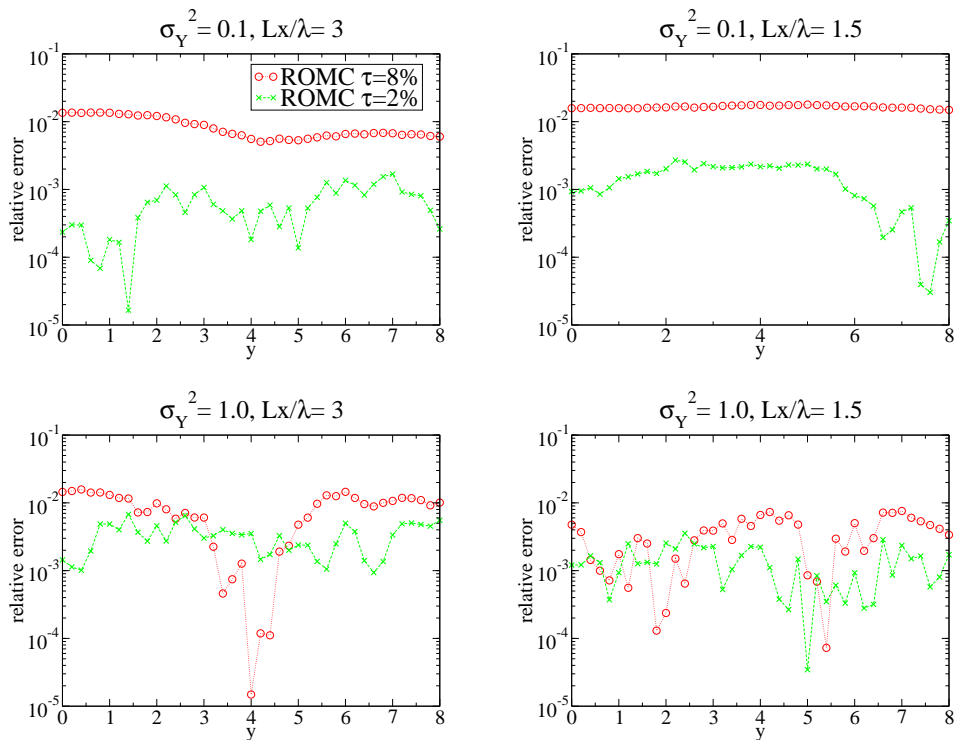

Fig. 6 Relative errors on the $10^{t h}$-percentile, $q_{10}$, of hydraulic head along the cross-section normal to the mean flow direction and passing through the domain centre.

be significant when we consider the complete range of the hydraulic heads $[0 \leq h \leq 10]$, resulting from the collection of MC realizations.

Results of comparable quality are obtained also for the remaining test cases (not shown).

\section{Conclusions}

Our work leads to the following major conclusions.

- The greedy algorithm is a suitable procedure to construct a reduced order model for the solution of steady-state groundwater flow in the presence of randomly distributed (Gaussian) log-transmissivities characterized by relatively low variance and highly persistent spatial distribution (i.e., large correlation length scales relative to the size of the domain). Our examples show that under these conditions the algorithm reduces the original FSM of dimension $n=3371$ to a $\mathrm{RM}$ of dimension $N_{B F}<50$ maintaining a high level of accuracy on each realization of hydraulic heads (see Fig. 2, for the test cases with $\sigma_{Y}^{2}<0.5$ and $L_{x} / \lambda<3$, with $\tau=2 \%$ ). In these cases, the procedure requires only a few FSM runs and is markedly advantageous with respect to the computational cost associated with the classical MC scheme $\left(10^{4} \mathrm{FSM}\right.$ runs in our case). An efficient and accurate reduction for 

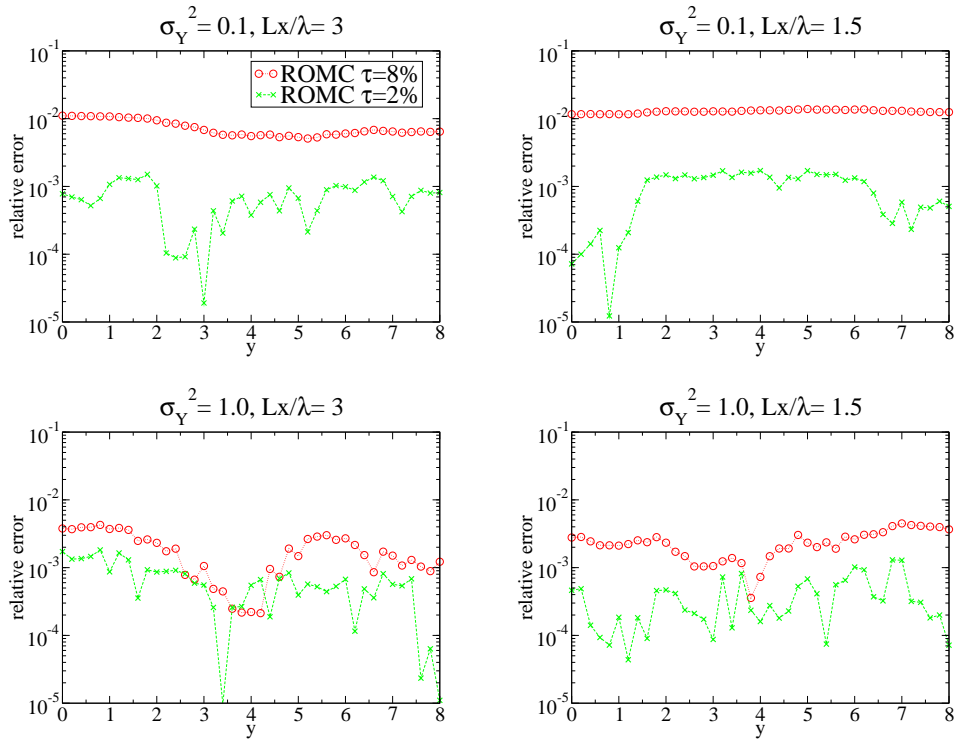

Fig. 7 Relative errors on the $90^{t h}$-percentile, $q_{90}$, of hydraulic heads along the cross-section normal to the mean flow direction and passing through the domain center.
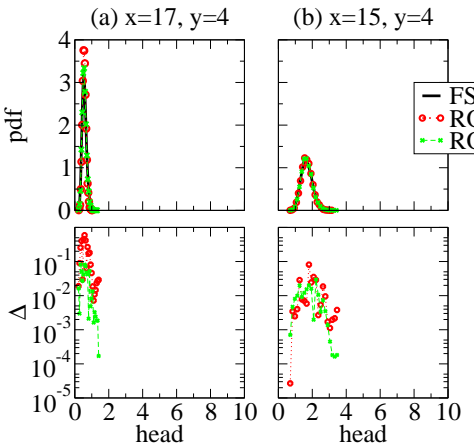

(c) $\mathrm{x}=9, \mathrm{y}=4$

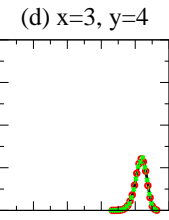

(e) $\mathrm{x}=1, \mathrm{y}=4$

Fig. 8 Comparison between the empirical pdfs of the FSM and ROMC head realizations at the five control points depicted in Fig. 1. Results for $L_{x} / \lambda=3$ and $\sigma_{Y}^{2}=0.1$ and error tolerances $\tau=8 \%, 2 \%$. The lower panels show the absolute differences $(\Delta)$ between the ROMC and the FSM results.

the configurations characterized by higher variances and smaller correlation lengths requires setting increased values of the error tolerance $\tau$, even though this compromises the accuracy on the reproduction of the head realizations and the empirical head distributions (Figs. 3, 4, and 5). 

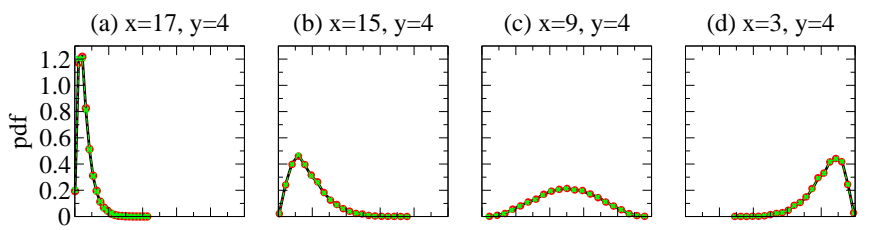

(e) $\mathrm{x}=1, \mathrm{y}=4$
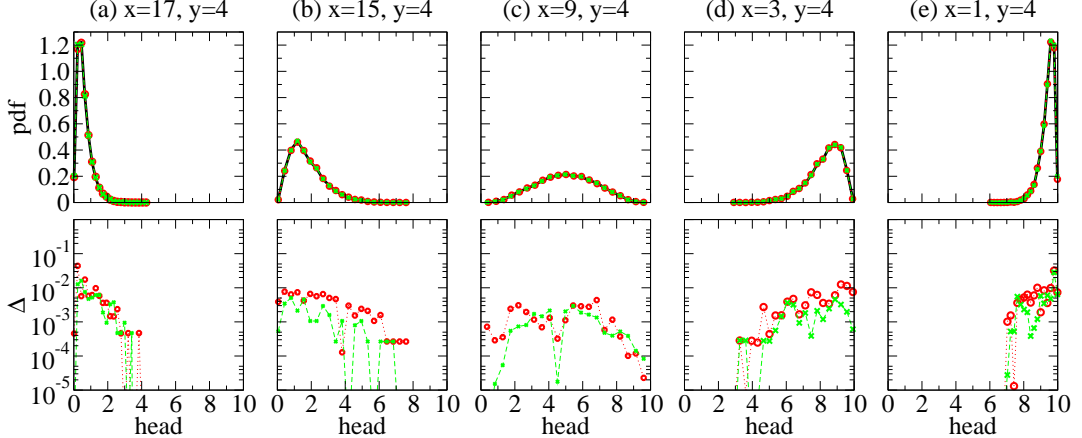

Fig. 9 Comparison between the complete empirical pdfs of the FSM and ROMC head realizations at the five control points depicted in Fig. 1. Results for $L_{x} / \lambda=3$ and $\sigma_{Y}^{2}=1.0$ and error tolerances $\tau=8 \%, 2 \%$. The lower panels show the absolute differences $(\Delta)$ between the ROMC and the FSM results.

- The rate of convergence of the largest error on the head realizations can be approximated as a power law function of the number of iterations of the greedy algorithm (Fig. 2, Table 1). This relationship between errors and number of iterations can be considered as an "a priori" criterion to establish the number of iterations of the greedy algorithm that are required to reach the desired error tolerance. Note that, since the implementation of the greedy algorithm with the residual-based estimation of the error may lead to a selection of snapshots that do not minimize the discrepancy between the FSM and ROMC, the convergence rate of the algorithm that employs residual based error estimations may be slower than the one presented here.

- The collection of hydraulic head realizations computed with the reduced order model is associated with an empirical probability distribution that well approximates the FSM-based sample distribution. We demonstrate this by showing comparisons of the spatial variance of heads (Figs. 3, 4, and 5), the spatial distribution of head percentiles along selected domain cross sections (Figs. 6 and 7) and the entire empirical pdf at selected grid nodes (Fig. 8 and 9) calculated through the FSM and RM set of realizations. As expected, the error on the ensemble statistics decreases with decreasing error tolerance in the greedy algorithm. It can be noted that, once the error tolerance $\tau$ is assigned, the accuracy of the estimation of the head statistics is not significantly deteriorated by changes in the geostatistical parameters that describe the random spatial distribution of the log-transmissivity. 
Acknowledgements Funding from MIUR (Italian Ministry of Education, Universities and

Research - PRIN2010-11; project "Innovative methods for water resources under hydroclimatic uncertainty scenarios"), from EU FP7 (project CLIMB "Climate Induced Changes on the Hydrology of Mediterranean Basins - Reducing Uncertainty and Quantifying Risk") and from the Fondazione CaRiPaRO (project "'Nonlinear Partial Differential Equations: models, analysis, and control-theoretic problems") is acknowledged.

\section{References}

1. G. Dagan. Flow and Transport in Porous Formations. Springer, New York, 1989.

2. D. Zhang. Stochastic Methods for Flow in Porous Media: Copying With Uncertainties. Academic Press, San Diego, CA, 2002.

3. A. Yustres, L. Asensio, J. Alonso, and V. Navarro. A review of Markov Chain Monte Carlo and information theory tools for inverse problems in subsurface flow. Comput. Geosci., 16(1):1-20, 2012. doi: 10.1007/s10596011-9249-z.

4. H. Moradkhani, C. M. DeChant, and S. Sorooshian. Evolution of ensemble data assimilation for uncertainty quantification using the particle filterMarkov chain Monte Carlo method. Water Resour. Res., 48(12):W12520, 2012. doi: 10.1029/2012WR012144.

5. A. Guadagnini and S. P. Neuman. Nonlocal and localized analyses of conditional mean steady state flow in bounded, randomly nonuniform domains: 1. Theory and computational approach. Water Resour. Res., 35 (10):2999-3018, 1999. doi: 10.1029/1999WR900160.

6. C. L. Winter, D. M. Tartakovsky, and A. Guadagnini. Moment differential equations for flow in highly heterogeneous porous media. Surv. Geophys., 24(1):81-106, 2003. doi: 10.1023/A:1022277418570.

7. M. Panzeri, M. Riva, A. Guadagnini, and S. P. Neuman. Data assimilation and parameter estimation via ensemble Kalman filter coupled with stochastic moment equations of transient groundwater flow. Water Resour. Res., 49, 2013. doi: 10.1002/wrcr.20113.

8. X. Sanchez-Vila, D. Fernandez-Garcia, and Guadagnini A. Conditional probability density functions of concentrations for mixing-controlled reactive transport in heterogeneous aquifers. Math. Geosci., 41:323-351, 2009. doi: $10.1007 / \mathrm{s} 11004-008-9204-2$.

9. D. M. Tartakovsky, M. Dentz, and P. C. Lichtner. Probability density functions for advective-reactive transport in porous media with uncertain reaction rates. Water Resour. Res., 45:W07414, 2009. doi: 10.1029/2008WR007383. 
10. M. Dentz and D. M. Tartakovsky. Probability density functions for passive scalars dispersed in random velocity fields. Geophys. Res. Lett., 45:L24406, 2010. doi: 10.1029/2010GL045748.

11. D. Venturi, D. M. Tartakovsky, A. M. Tartakovsky, and G. E. Karniadakis. Exact pdf equations and closure approximations for advective-reactive transport. J. Comp. Phys., 243:323-343, 2013. doi: 10.1016/j.jcp.2013.03.001.

12. F. Ballio and A. Guadagnini. Convergence assessment of numerical Monte Carlo simulations in groundwater hydrology. Water Resour. Res., 40: W04603, 2004. doi: 10.1029/2003WR002876.

13. D. Pasetto, A. Guadagnini, and M. Putti. POD-based Monte Carlo approach for the solution of regional scale groundwater flow driven by randomly distributed recharge. Adv. Water Resources, 34(11):1450-1463, 2011. doi: 10.1016/j.advwatres.2011.07.003.

14. D. Zhang and Z. Lu. An efficient, high-order perturbation approach for flow in random porous media via Karhunen-Loève and polynomial expansions. J. Comp. Phys., 194(2):773 - 794, $2004 . \quad$ doi: 10.1016/j.jcp.2003.09.015.

15. S. Poles and A. Lovison. A polynomial chaos approach to robust multiobjective optimization. In Deb, K., et al., editor, Hybrid and Robust Approaches to Multiobjective Optimization, number 09041 in Dagstuhl Seminar Proceedings. Schloss Dagstuhl - Leibniz-Zentrum fuer Informatik, Germany, Dagstuhl, Germany, 2009.

16. S. Oladyshkin, H. Class, R. Helmig, and W. Nowak. An integrative approach to robust design and probabilistic risk assessment for CO2 storage in geological formations. Comput. Geosci., 15(3):565-577, 2011. doi: 10.1007/s10596-011-9224-8.

17. L. Formaggia, A. Guadagnini, I. Imperiali, V. Lever, G. Porta, M. Riva, A. Scotti, and L. Tamellini. Global sensitivity analysis through polynomial chaos expansion of a basin-scale geochemical compaction model. Comput. Geosci., 17:25-42, 2013. doi: 10.1007/s10596-012-9311-5.

18. H. Li and D. Zhang. Probabilistic collocation method for flow in porous media: Comparisons with other stochastic methods. Water Resour. Res., 43:W09409, 2007. doi: 10.1029/2006WR005673.

19. K. Kunisch and S. Volkwein. Galerkin proper orthogonal decomposition methods for a general equation in fluid dynamics. SIAM J. Num. Anal., 40:492-515, 2002. doi: 10.1137/S0036142900382612.

20. A. J. Siade, M. Putti, and W. W.-G. Yeh. Snapshot selection for groundwater model reduction using proper orthogonal decomposition. Water Resour. Res., 46:W08539, 2010. doi: 10.1029/2009WR008792.

21. M. A. Grepl and A. T. Patera. A posteriori error bounds for reduced-basis approximations of parametrized parabolic partial differential equations. ESAIM-Math. Model. Num., 39(1):157-181, 2005. doi: 10.1051/m2an:2005006.

22. A. Quarteroni, G. Rozza, and A. Manzoni. Certified reduced basis approximation for parametrized partial differential equations and applications. 
Math. Indust., 1(1):1-49, 2011. doi: 10.1186/2190-5983-1-3.

23. A. J. Siade, M. Putti, and W. W.-G. Yeh. Reduced order parameter estimation using quasilinearization and quadratic programming. Water Resour. Res., 48:W06502, 2012. doi: 10.1029/2011WR011471.

24. M. P. Kaleta, R. G. Hanea, A. W. Heemink, and J-D. Jansen. Modelreduced gradient-based history matching. Comput. Geosci., 15(1):135153, 2011. doi: $10.1007 / \mathrm{s} 10596-010-9203-5$.

25. J. F. M. van Doren, R. Markovinović, and J-D. Jansen. Reduced-order optimal control of water flooding using proper orthogonal decomposition. Comput. Geosci., 10(1):137-158, 2006. doi: 10.1007/s10596-005-9014-2.

26. D. Pasetto, M. Putti, and W. W-G. Yeh. A reduced order model for groundwater flow equation with random hydraulic conductivity: application to Monte Carlo methods. Water Resour. Res., 49:1-14, 2013. doi: 10.1002 /wrcr. 20136.

27. F. Müller, P. Jenny, and D. W. Meyer. Multilevel Monte Carlo for two phase flow and Buckley-Leverett transport in random heterogeneous porous media. J. Comp. Phys., 2013. doi: 10.1016/j.jcp.2013.03.023.

28. C. Lieberman, K. Willcox, and O. Ghattas. Parameter and state model reduction for large-scale statistical inverse problems. SIAM J. Sci. Comput., 32(5):2523-2542, 2010. doi: 10.2307/2236101.

29. A. Bellin and Y. Rubin. HYDRO_GEN: A spatially distributed random field generator for correlated properties. Stoch. Hydrol. Hydraul., 10(4): 253-278, 1996. doi: 10.1007/BF01581869. 